\title{
Onset of superradiant instabilities in rotating spacetimes of exotic compact objects
}

\author{
Shahar Hod \\ The Ruppin Academic Center, \\ Emeq Hefer 40250, Israel \\ The Hadassah Academic College, \\ Jerusalem 91010, Israel \\ E-mail: shaharhod@gmail.com
}

ABSTRACT: Exotic compact objects, horizonless spacetimes with reflective properties, have intriguingly been suggested by some quantum-gravity models as alternatives to classical black-hole spacetimes. A remarkable feature of spinning horizonless compact objects with reflective boundary conditions is the existence of a discrete set of critical surface radii, $\left\{r_{\mathrm{c}}(\bar{a} ; n)\right\}_{n=1}^{n=\infty}$, which can support spatially regular static (marginally-stable) scalar field configurations (here $\bar{a} \equiv J / M^{2}$ is the dimensionless angular momentum of the exotic compact object). Interestingly, the outermost critical radius $r_{\mathrm{c}}^{\max } \equiv \max _{n}\left\{r_{\mathrm{c}}(\bar{a} ; n)\right\}$ marks the boundary between stable and unstable exotic compact objects: spinning objects whose reflecting surfaces are situated in the region $r_{\mathrm{c}}>r_{\mathrm{c}}^{\max }(\bar{a})$ are stable, whereas spinning objects whose reflecting surfaces are situated in the region $r_{\mathrm{c}}<r_{\mathrm{c}}^{\max }(\bar{a})$ are superradiantly unstable to scalar perturbation modes. In the present paper we use analytical techniques in order to explore the physical properties of the critical (marginally-stable) spinning exotic compact objects. In particular, we derive a remarkably compact analytical formula for the discrete spectrum $\left\{r_{\mathrm{c}}^{\max }(\bar{a})\right\}$ of critical radii which characterize the marginally-stable exotic compact objects. We explicitly demonstrate that the analytically derived resonance spectrum agrees remarkably well with numerical results that recently appeared in the physics literature.

Keywords: Black Holes, Classical Theories of Gravity, Models of Quantum Gravity

ARXIV EPRINT: 1704.05856 


\section{Contents}

1 Introduction $\quad 1$

2 Description of the system 2

3 The resonance conditions of the marginally-stable spinning exotic compact objects 4

4 The discrete resonance spectra of the marginally-stable spinning exotic $\begin{array}{ll}\text { compact objects } & 6\end{array}$

5 Resonance spectra for highly-compact spinning exotic objects $\quad 7$

$\begin{array}{lll}5.1 \text { An analytical treatment } & 7\end{array}$

5.2 Numerical confirmation 8

6 Analytical vs. former numerical results 10

$\begin{array}{lll}7 & \text { Summary and discussion } & 10\end{array}$

\section{Introduction}

Black holes are certainly the most important prediction of general relativity, Einstein's classical theory of gravity. These fundamental objects are characterized by curved spacetime geometries with absorbing boundary conditions (event horizons). Intriguingly, however, some researchers (see [1-13] and references therein) have recently examined the possibility that horizonless compact objects may serve as exotic alternatives to the familiar (classical) black-hole spacetimes.

It is certainly of physical importance to explore the (in)stability properties of these horizonless exotic compact objects. In a very important work, Maggio, Pani, and Ferrari [13] have recently demonstrated numerically that, due to the physical mechanism of superradiant amplification [14, 15], horizonless spinning compact objects with reflective boundary conditions and ergoregions may become unstable to scalar perturbation modes. ${ }^{1}$ Intriguingly, the results presented in [13] have revealed the important fact that the ergoregion instability shuts down if the quantum reflective surface of the spinning exotic compact object is located far enough from the would-be classical black-hole horizon. This interesting and highly important numerical finding implies, in particular, that there exists

\footnotetext{
${ }^{1}$ It is worth noting that the superradiant instability phenomenon observed in [15] is also expected to characterize the dynamics of other bosonic (integer-spin) fields in the curved spacetimes of these horizonless spinning exotic compact objects.
} 
a unique family of critical (marginally-stable) exotic compact objects which determine the boundary between stable and unstable horizonless spinning configurations.

In the present paper we shall use analytical techniques in order to explore the physical properties of the critical (marginally-stable) exotic compact objects. In particular, below we shall explicitly prove that horizonless spinning compact objects with reflective boundary conditions are characterized by the existence of a discrete set $\left\{r_{\mathrm{c}}(\bar{a} ; n)\right\}_{n=1}^{n=\infty}$ of critical surface radii that can support spatially regular static (marginally-stable) massless scalar field configurations $[16-45]^{2}$ (here $\bar{a}$ is the dimensionless angular momentum of the exotic compact object, see eq. (3.2) below).

It should be emphasized that the physical significance of the outermost (largest) radius $r_{\mathrm{c}}^{\max }(\bar{a}) \equiv \max _{n}\left\{r_{\mathrm{c}}(\bar{a} ; n)\right\}$ stems from the fact that, for a given value of the dimensionless rotation parameter $\bar{a}$, this unique critical radius marks the boundary between stable and unstable spinning exotic compact configurations. In particular, horizonless compact objects of rotation parameter $\bar{a}$ whose reflecting surfaces are characterized by the inequality $r_{\mathrm{c}}<$ $r_{\mathrm{c}}^{\max }(\bar{a})$ are superradiantly unstable to scalar perturbation modes, whereas horizonless compact objects whose reflecting surfaces are characterized by the inequality $r_{\mathrm{c}}>r_{\mathrm{c}}^{\max }(\bar{a})$ are stable.

The main goal of the present paper is to determine analytically the discrete spectrum $\left\{r_{\mathrm{c}}(\bar{a} ; n)\right\}_{n=1}^{n=\infty}$ of radii which characterize the horizonless spinning exotic compact objects that can support the spatially regular static (marginally-stable) scalar field configurations. In particular, below we shall derive a remarkably compact analytical formula for the critical (outermost) radii $\left\{r_{\mathrm{c}}^{\max }(\bar{a})\right\}$ of the exotic compact objects that mark the boundary between stable and unstable horizonless spinning configurations.

\section{Description of the system}

We shall analyze the physical properties of horizonless spinning compact objects with reflective surfaces which are linearly coupled to massless scalar fields. Following the interesting work of Maggio, Pani, and Ferrari [13] (see also [1-12]), we shall study exotic compact objects which are characterized by curved geometries that modify the spinning Kerr spacetime only at some microscopic scale around the would-be classical horizon. In particular, we shall assume, as in [13], that the Kerr line element [46] $]^{3,4,5}$

$$
d s^{2}=-\frac{\Delta}{\rho^{2}}\left(d t-a \sin ^{2} \theta d \phi\right)^{2}+\frac{\rho^{2}}{\Delta} d r^{2}+\rho^{2} d \theta^{2}+\frac{\sin ^{2} \theta}{\rho^{2}}\left[a d t-\left(r^{2}+a^{2}\right) d \phi\right]^{2} \text { for } r>r_{\mathrm{c}}
$$

\footnotetext{
${ }^{2}$ It is worth mentioning that spinning Kerr black holes, as opposed to the horizonless spinning compact objects that we study in the present paper, cannot support static spatially regular massless scalar fields in their exterior regions. However, as recently demonstrated in [16-45], spinning black holes can support stationary (rather than static) massive scalar field configurations.

${ }^{3}$ We shall use natural units in which $G=c=\hbar=1$.

${ }^{4}$ Here we have used the familiar Boyer-Lindquist coordinates $(t, r, \theta, \phi)$.

${ }^{5}$ Following the physically interesting model of the exotic compact objects studied in [13], we shall assume that the energy and angular momentum of the reflective surface are negligible.
} 
with $\Delta \equiv r^{2}-2 M r+a^{2}$ and $\rho^{2} \equiv r^{2}+a^{2} \cos ^{2} \theta$, describes the external spacetime of the spinning exotic compact object of mass $M$, angular momentum $J=M a$, and radius $r_{\mathrm{c}}$. In addition, below we shall assume that the radius of the exotic compact object is characterized by the relation $[47-49]^{6}$

$$
z_{\mathrm{c}} \equiv \frac{r_{\mathrm{c}}-r_{+}}{r_{+}} \ll 1
$$

where

$$
r_{ \pm}=M \pm\left(M^{2}-a^{2}\right)^{1 / 2}
$$

are the radii of the would-be classical horizons. The small $-z_{\mathrm{c}}$ regime (2.2) corresponds to the physically interesting family of horizonless exotic compact objects whose quantum reflective surfaces are located a microscopic distance above the would-be classical black-hole horizons [13] (see also [1-12]).

The dynamics of the linearized massless scalar field in the spinning spacetime of the exotic compact object is governed by the Klein-Gordon wave equation [50-52]

$$
\nabla^{\nu} \nabla_{\nu} \Psi=0 .
$$

Substituting into (2.4) the metric components of the curved line element (2.1), which characterizes the exterior spacetime of the spinning exotic compact object, and using the scalar field decomposition $[50-52]^{7}$

$$
\Psi=\sum_{l, m} e^{i m \phi} S_{l m}(\theta ; a \omega) R_{l m}(r ; M, a, \omega) e^{-i \omega t},
$$

one finds that the radial part $R_{l m}(r ; M, a, \omega)$ of the scalar eigenfunction is determined by the ordinary differential equation $[50-52]$

$$
\Delta \frac{d}{d r}\left(\Delta \frac{d R_{l m}}{d r}\right)+\left\{\left[\omega\left(r^{2}+a^{2}\right)-m a\right]^{2}+\Delta\left(2 m a \omega-K_{l m}\right)\right\} R_{l m}=0 .
$$

Here the frequency-dependent parameter $K_{l m}(a \omega)$ is the characteristic eigenvalue of the spatially regular angular eigenfunction $S_{l m}(\theta ; a \omega)$, which is determined by the familiar spheroidal differential equation [50-63]

$$
\frac{1}{\sin \theta} \frac{d}{\theta}\left(\sin \theta \frac{d S_{l m}}{d \theta}\right)+\left(K_{l m}-a^{2} \omega^{2}+a^{2} \omega^{2} \cos ^{2} \theta-\frac{m^{2}}{\sin ^{2} \theta}\right) S_{l m}=0 .
$$

For later purposes we note that, in the small frequency $a \omega \ll 1$ regime, the angular eigenvalues of the spheroidal scalar eigenfunctions can be expanded in the from

$$
K_{l m}-a^{2} \omega^{2}=l(l+1)+\sum_{k=1}^{\infty} c_{k}(a \omega)^{2 k},
$$

where the frequency-independent coefficients $\left\{c_{k}(l, m)\right\}$ are given in [59].

\footnotetext{
${ }^{6}$ It is important to stress the fact that the assumption made in the physical model of [13], according to which the exterior curved spacetimes of the spinning exotic objects are described by the familiar Kerr line element (2.1), is a non-trivial one. As discussed in [13] (see also [47-49]), this assumption is expected to be valid in the physically interesting regime $z_{\mathrm{c}} \ll 1$ of exotic compact objects whose reflecting quantum membranes are located a microscopic distance above the would-be classical horizons.

${ }^{7}$ The physical parameters $\{\omega, l, m\}$ are respectively the proper frequency, the spheroidal harmonic index, and the azimuthal harmonic index (with $-l \leq m \leq l$ ) which characterize the massless scalar field mode.
} 
Following the physically interesting model of the spinning exotic objects studied numerically in [13], we shall assume that the horizonless compact objects are characterized by (Dirichlet or Neumann) reflecting boundary conditions: ${ }^{8}$

$$
\begin{cases}R\left(r=r_{\mathrm{c}}\right)=0 & \text { Dirichlet B.C. } \\ d R\left(r=r_{\mathrm{c}}\right) / d r=0 & \text { Neumann B.C. }\end{cases}
$$

In addition, the marginally-stable (static) scalar modes that we shall analyze in the present paper are characterized by spatially regular (asymptotically decaying) eigenfunctions:

$$
R(r \rightarrow \infty) \rightarrow 0
$$

\section{The resonance conditions of the marginally-stable spinning exotic com- pact objects}

The composed horizonless-spinning-object-massless-scalar-field system is characterized by the existence of a unique family of marginally-stable (static) resonances which mark the onset of superradiant instabilities in the curved spinning spacetime. These physically interesting critical field modes are characterized by the simple property

$$
\omega=0 \text {. }
$$

It is worth noting that the intriguing and well-studied physical mechanism of superradiance $[14,15,64-68]$ allows integer-spin (bosonic) fields to extract rotational energy and angular momentum from spinning objects. In particular, an orbiting scalar field mode of azimuthal harmonic index $m$ interacting with a central spinning object of angular velocity $\Omega$ can be amplified in the bounded frequency regime $0<\omega_{\text {field }}<m \Omega[14,15,64-68]$. As emphasized above, the composed horizonless-spinning-object-massless-scalar-field configurations that we shall analyze in the present paper are characterized by the relation $\omega_{\text {field }}=0$ (see eq. (3.1)). Thus, these marginally-stable composed configurations sit on the boundary of the superradiant regime.

The set of equations (2.6)-(3.1) determines two discrete spectra of radii, $\left\{r_{\mathrm{c}}^{\text {Dirichlet }}\right.$ $(\bar{a}, l, m ; n)\}$ and $\left\{r_{\mathrm{c}}^{\text {Neumann }}(\bar{a}, l, m ; n)\right\}$, which, for a given value

$$
\bar{a} \equiv \frac{J}{M^{2}}
$$

of the dimensionless angular momentum parameter, characterize the critical (marginallystable) spinning exotic compact objects. Interestingly, as we shall explicitly show in the present section, the characteristic radial equation (2.6) of the massless scalar fields in the curved spacetimes of the spinning exotic compact objects is amenable to an analytical treatment for the marginally-stable static modes.

Substituting into eq. (2.6) $\omega=0$ and

$$
R(x)=x^{-i \alpha}(1-x)^{l+1} F(x),
$$

\footnotetext{
${ }^{8}$ For brevity, we shall henceforth omit the angular harmonic indices $(l, m)$ of the massless scalar field mode.
} 
where

$$
x \equiv \frac{r-r_{+}}{r-r_{-}} ; \quad \alpha \equiv \frac{m a}{r_{+}-r_{-}},
$$

one obtains the characteristic radial equation ${ }^{9}$

$$
x(1-x) \frac{d^{2} F}{d x^{2}}+\{(1-2 i \alpha)-[1+2(l+1)-2 i \alpha] x\} \frac{d F}{d x}-\left[(l+1)^{2}-2 i \alpha(l+1)\right] F=0 .
$$

The general mathematical solution of the radial differential equation (3.5) is given by $[59,69]$

$F(x)=A \cdot{ }_{2} F_{1}(l+1-2 i \alpha, l+1 ; 2 l+2 ; 1-x)+B \cdot(1-x)^{-2 l-1}{ }_{2} F_{1}(-l-2 i \alpha,-l ;-2 l ; 1-x)$,

where ${ }_{2} F_{1}(a, b ; c ; z)$ is the hypergeometric function and $\{A, B\}$ are normalization constants. Substituting (3.6) into (3.3), one obtains the expression

$$
\begin{gathered}
R(x)=x^{-i \alpha}\left[A \cdot(1-x)^{l+1}{ }_{2} F_{1}(l+1-2 i \alpha, l+1 ; 2 l+2 ; 1-x)\right. \\
\left.+B \cdot(1-x)^{-l}{ }_{2} F_{1}(-l-2 i \alpha,-l ;-2 l ; 1-x)\right]
\end{gathered}
$$

for the radial scalar eigenfunction. Using the characteristic property (see eq. (15.1.1) of [59])

$$
{ }_{2} F_{1}(a, b ; c ; z \rightarrow 0) \rightarrow 1
$$

of the hypergeometric function, one finds from (3.7) the asymptotic $r \rightarrow \infty\left(x \rightarrow 1^{-}\right.$, see eq. (3.4)) behavior

$$
R(x \rightarrow 1)=A \cdot(1-x)^{l+1}+B \cdot(1-x)^{-l}
$$

of the radial eigenfunction. A physically acceptable (finite) solution at spatial infinity $\left(r \rightarrow \infty\right.$, or equivalently $\left.x \rightarrow 1^{-}\right)$requires

$$
B=0 .
$$

We therefore conclude that the marginally-stable (static) resonances of the massless scalar fields in the curved spacetimes of the horizonless spinning exotic compact objects are characterized by the radial eigenfunction

$$
R(x)=A \cdot x^{-i \alpha}(1-x)^{l+1}{ }_{2} F_{1}(l+1-2 i \alpha, l+1 ; 2 l+2 ; 1-x) .
$$

Taking cognizance of the boundary conditions (2.9), which characterize the horizonless reflecting compact objects, one deduces that the compact resonance equations

$$
{ }_{2} F_{1}\left(l+1-2 i \alpha, l+1 ; 2 l+2 ; 1-x_{\mathrm{c}}\right)=0 \quad \text { for Dirichlet B.C. }
$$

and

$$
\frac{d}{d x}\left[x^{-i \alpha}(1-x)^{l+1}{ }_{2} F_{1}(l+1-2 i \alpha, l+1 ; 2 l+2 ; 1-x)\right]_{x=x_{\mathrm{c}}}=0 \quad \text { for Neumann B.C. }
$$

determine the discrete spectra of dimensionless critical radii $\left\{x_{\mathrm{c}}(\bar{a}, l, m ; n)\right\}$ which characterize the marginally-stable exotic compact objects.

\footnotetext{
${ }^{9}$ Here we have used the relation $K_{l m}(a \omega=0)=l(l+1)$ (see eq. (2.8)).
} 


\begin{tabular}{|c|c|c|c|c|c|c|}
\hline Boundary conditions & $z_{\mathrm{c}}^{\max }(\bar{a}=0.3)$ & $z_{\mathrm{c}}^{\max }(\bar{a}=0.5)$ & $z_{\mathrm{c}}^{\max }(\bar{a}=0.7)$ & $z_{\mathrm{c}}^{\max }(\bar{a}=0.9)$ & $z_{\mathrm{c}}^{\max }(\bar{a}=0.99)$ & $z_{\mathrm{c}}^{\max }(\bar{a}=0.999)$ \\
\hline Dirichlet & $2.960 \times 10^{-10}$ & $2.842 \times 10^{-6}$ & $2.783 \times 10^{-4}$ & $1.007 \times 10^{-2}$ & $9.625 \times 10^{-2}$ & $1.730 \times 10^{-1}$ \\
Neumann & $6.455 \times 10^{-6}$ & $6.662 \times 10^{-4}$ & $7.417 \times 10^{-3}$ & $5.432 \times 10^{-2}$ & $2.105 \times 10^{-1}$ & $3.078 \times 10^{-1}$ \\
\hline
\end{tabular}

Table 1. Marginally-stable spinning exotic compact objects. We display, for various values of the dimensionless angular momentum parameter $\bar{a}$, the largest dimensionless radii $z_{\mathrm{c}}^{\max }(\bar{a}) \equiv\left(r_{\mathrm{c}}^{\max }-\right.$ $\left.r_{+}\right) / r_{+}$(see eqs. (4.1) and (4.2)) of the horizonless reflecting compact objects that can support the spatially regular static scalar field configurations with $l=m=1$. One finds that the critical radii $\left\{z_{\mathrm{c}}^{\max }(\bar{a})\right\}$, which characterize the marginally-stable spinning exotic compact objects, are a monotonically increasing function of the dimensionless angular momentum parameter $\bar{a}$.

\section{The discrete resonance spectra of the marginally-stable spinning exotic compact objects}

The analytically derived resonance equations (3.12) and (3.13), which characterize the unique families of horizonless exotic compact objects that can support the static (marginally-stable) massless scalar field configurations, can easily be solved numerically. Interestingly, one finds that, for given physical parameters $\{\bar{a}, l, m\}$ of the composed spinningexotic-compact-object-massless-scalar-field system, there exists a discrete set of critical radii,

$$
\cdots r_{\mathrm{c}}(n=3)<r_{\mathrm{c}}(n=2)<r_{\mathrm{c}}(n=1) \equiv r_{\mathrm{c}}^{\max }(\bar{a}, l, m),
$$

which can support the spatially regular static (marginally-stable) scalar field resonances. ${ }^{10}$

In table 1 we display, for various values of the dimensionless angular momentum parameter $\bar{a}$, the largest (outermost) dimensionless radii ${ }^{11}$

$$
z_{\mathrm{c}}^{\max }(\bar{a}, l, m) \equiv \frac{r_{\mathrm{c}}^{\max }-r_{+}}{r_{+}}
$$

of the horizonless spinning compact objects that can support the marginally-stable massless scalar field configurations. From the data presented in table 1 one learns that, for fixed values of the scalar angular harmonic indices, the critical radii $z_{\mathrm{c}}^{\max }(\bar{a})$ (and thus also $r_{\mathrm{c}}^{\max }(\bar{a})$ ) which characterize the marginally-stable spinning exotic compact objects are a monotonically increasing function of the dimensionless angular momentum parameter $\bar{a}$.

It is worth emphasizing again that the physical significance of the critical reflecting radius $r_{\mathrm{c}}^{\max }(\bar{a})$ stems from the fact that, for a given value of the dimensionless angular

\footnotetext{
${ }^{10} \mathrm{It}$ is worth noting that the characteristic radial scalar eigenfunction (3.11) has an infinite number of nodes in the $x_{\mathrm{c}} \rightarrow 0\left(r_{\mathrm{c}} \rightarrow r_{+}\right)$limit. For finite values of $x_{\mathrm{c}}$, the radial eigenfunction (3.11) has a finite number of nodes with the property $x \geq x_{\mathrm{c}}$ (that is, on and outside the reflecting surface of the exotic compact object). In particular, for Dirichlet boundary conditions, the resonance parameter $n$ counts the number of nodes (including the node at the surface of the exotic compact object) of the radial scalar eigenfunction (3.11). Likewise, for Neumann boundary conditions, the resonance parameter $n$ counts the number of extremum points (including the one at the surface of the exotic compact object) of the radial scalar eigenfunction (3.11), whereas the number of field nodes outside the surface of the compact spinning object is given by $n-1$ in this case.

${ }^{11}$ Note that the dimensionless coordinate $z_{\mathrm{c}}$, which characterizes the radius of the horizonless reflecting exotic compact object, can be expressed in the form $z_{\mathrm{c}}=\frac{x_{\mathrm{c}}}{1-x_{\mathrm{c}}} \cdot \frac{r_{+}-r_{-}}{r_{+}}$(see eqs. (3.4) and (4.2)).
} 


\begin{tabular}{|c|c|c|c|c|c|c|}
\hline Boundary conditions & $z_{\mathrm{c}}^{\max }(l=1)$ & $z_{\mathrm{c}}^{\max }(l=2)$ & $z_{\mathrm{c}}^{\max }(l=3)$ & $z_{\mathrm{c}}^{\max }(l=4)$ & $z_{\mathrm{c}}^{\max }(l=5)$ & $z_{\mathrm{c}}^{\max }(l=6)$ \\
\hline Dirichlet & $2.842 \times 10^{-6}$ & $3.689 \times 10^{-4}$ & $1.788 \times 10^{-3}$ & $3.948 \times 10^{-3}$ & $6.395 \times 10^{-3}$ & $8.870 \times 10^{-3}$ \\
Neumann & $6.662 \times 10^{-4}$ & $6.141 \times 10^{-3}$ & $1.268 \times 10^{-2}$ & $1.832 \times 10^{-2}$ & $2.297 \times 10^{-2}$ & $2.680 \times 10^{-2}$ \\
\hline
\end{tabular}

Table 2. Marginally-stable spinning exotic compact objects. We present the largest dimensionless radii $z_{\mathrm{c}}^{\max }(l) \equiv\left(r_{\mathrm{c}}^{\max }-r_{+}\right) / r_{+}$(see eqs. (4.1) and (4.2)) of the horizonless reflecting compact objects with dimensionless angular momentum parameter $\bar{a}=0.5$ that can support static equatorial $(l=m)$ scalar field configurations. One finds that the critical radii $\left\{z_{\mathrm{c}}^{\max }(l)\right\}$, which characterize the marginally-stable spinning exotic compact objects, are a monotonically increasing function of the scalar harmonic index $l$.

momentum parameter $\bar{a}$, this supporting radius corresponds to a marginally-stable spinning object which marks the onset of superradiant instabilities in the composed exoticcompact-object-massless-scalar-field system. In particular, as nicely demonstrated numerically in [13], spinning compact objects of dimensionless angular momentum $\bar{a}$ whose reflecting surfaces are located in the region $r_{\mathrm{c}}>r_{\mathrm{c}}^{\max }(\bar{a})$ are stable, whereas spinning objects whose reflecting surfaces are located in the region $r_{\mathrm{c}}<r_{\mathrm{c}}^{\max }(\bar{a})$ are superradiantly unstable to scalar perturbation modes.

In table 2 we display, for various equatorial $(l=m)$ massless scalar field modes, the critical (largest) dimensionless radii $z_{\mathrm{c}}^{\max }(l)$ (see eq. (4.2)) of the horizonless spinning compact objects that can support the static (marginally-stable) scalar field resonances. From the data presented in table 2 one learns that, for a fixed value of the dimensionless angular momentum parameter $\bar{a}$, the dimensionless critical radii $z_{\mathrm{c}}^{\max }(l)$ (and thus also $\left.r_{\mathrm{c}}^{\max }(l)\right)$ which characterize the marginally-stable spinning exotic compact objects are a monotonically increasing function of the scalar harmonic index $l$.

\section{Resonance spectra for highly-compact spinning exotic objects}

\subsection{An analytical treatment}

In the present section we shall explicitly prove that the resonance conditions (3.12) and (3.13), which respectively determine the dimensionless discrete radii $\left\{x_{\mathrm{c}}^{\text {Dirichlet }}(\bar{a}, l, m ; n)\right\}$ and $\left\{x_{\mathrm{c}}^{\text {Neumann }}(\bar{a}, l, m ; n)\right\}$ of the marginally-stable horizonless spinning exotic compact objects, can be solved analytically in the physically interesting regime

$$
x_{\mathrm{c}} \ll 1 .
$$

As emphasized above, the small- $x_{\mathrm{c}}$ regime (5.1) corresponds to the physically interesting family of highly compact exotic objects whose quantum reflective surfaces are located very near the would-be classical black-hole horizons. It is worth mentioning, in particular, that the strong inequality (5.1) characterizes the horizonless spinning exotic compact objects that were recently studied numerically in the interesting work of Maggio, Pani, and Ferrari $[13]$ (see also [1-12]). 
Using eq. (15.3.6) of [59], one can express the characteristic radial scalar eigenfunction (3.11) in the form ${ }^{12}$

$$
\begin{aligned}
R(x)=A \cdot \frac{(2 l+1) !}{l !}(1-x)^{l+1}[ & \frac{\Gamma(2 i \alpha)}{\Gamma(l+1+2 i \alpha)} x^{-i \alpha}{ }_{2} F_{1}(l+1-2 i \alpha, l+1 ; 1-2 i \alpha ; x) \\
& \left.+\frac{\Gamma(-2 i \alpha)}{\Gamma(l+1-2 i \alpha)} x^{i \alpha}{ }_{2} F_{1}(l+1+2 i \alpha, l+1 ; 1+2 i \alpha ; x)\right] .
\end{aligned}
$$

Taking cognizance of the characteristic asymptotic property (3.8) of the hypergeometric functions, one finds from (5.2) the small- $x$ behavior

$$
R(x \ll 1)=A \frac{(2 l+1) !}{l !}(1-x)^{l+1}\left[\frac{\Gamma(2 i \alpha)}{\Gamma(l+1+2 i \alpha)} x^{-i \alpha}+\frac{\Gamma(-2 i \alpha)}{\Gamma(l+1-2 i \alpha)} x^{i \alpha}\right] \cdot[1+O(x)]
$$

of the radial scalar eigenfunction.

Using the characteristic small- $x$ spatial behavior (5.3) of the radial scalar eigenfunction, one can express the Dirichlet and Neumann resonance equations (3.12) and (3.13) in the remarkably compact form

$$
x^{2 i \alpha}=\mp \frac{\Gamma(2 i \alpha) \Gamma(l+1-2 i \alpha)}{\Gamma(-2 i \alpha) \Gamma(l+1+2 i \alpha)},
$$

where the upper/lower signs in (5.4) refer respectively to the reflecting Dirichlet/Neumann boundary conditions. From the resonance conditions (5.4) one finally finds the compact analytical formulas ${ }^{13,14}$

$$
x_{\mathrm{c}}^{\text {Dirichlet }}(n)=e^{-\pi\left(n+\frac{1}{2}\right) / \alpha}\left[\frac{\Gamma(2 i \alpha) \Gamma(l+1-2 i \alpha)}{\Gamma(-2 i \alpha) \Gamma(l+1+2 i \alpha)}\right]^{1 / 2 i \alpha} ; \quad n \in \mathbb{Z}
$$

and

$$
x_{\mathrm{c}}^{\text {Neumann }}(n)=e^{-\pi n / \alpha}\left[\frac{\Gamma(2 i \alpha) \Gamma(l+1-2 i \alpha)}{\Gamma(-2 i \alpha) \Gamma(l+1+2 i \alpha)}\right]^{1 / 2 i \alpha} ; n \in \mathbb{Z}
$$

for the discrete families $\left\{x_{\mathrm{c}}^{\text {Dirichlet }}(n), x_{\mathrm{c}}^{\text {Neumann }}(n)\right\}$ of dimensionless critical radii which characterize the horizonless spinning compact objects that can support the spatially regular static (marginally-stable) massless scalar field resonances.

\subsection{Numerical confirmation}

It is physically important to verify the validity of the analytically derived resonance spectra (5.5) and (5.6) for the characteristic discrete radii of the highly compact $\left(x_{\mathrm{c}} \ll 1\right)$ spinning exotic objects that can support the static (marginally-stable) scalar field configurations. In tables 3 and 4 we display the dimensionless radii $z_{\mathrm{c}}^{\text {analytical }}(n) \equiv\left[r_{\mathrm{c}}(n)-r_{+}\right] / r_{+}$

\footnotetext{
${ }^{12}$ Here we have used eq. (6.1.15) of [59].

${ }^{13}$ Here we have used the relation $1=e^{-2 i \pi n}$, where $n$ is an integer. This dimensionless resonance parameter characterizes the composed spinning-exotic-compact-object-massless-scalar-field configurations.

${ }^{14}$ Taking cognizance of eq. (6.1.23) of [59], one finds the relations $\Gamma(2 i \alpha) / \Gamma(-2 i \alpha)=e^{i \phi_{1}}$ and $\Gamma(l+1-$ $2 i \alpha) / \Gamma(l+1+2 i \alpha)=e^{i \phi_{2}}$, where $\left\{\phi_{1}, \phi_{2}\right\} \in \mathbb{R}$. These characteristic relations imply, in particular, that $\left\{x_{\mathrm{c}}^{\text {Dirichlet }}(n), x_{\mathrm{c}}^{\text {Neumann }}(n)\right\} \in \mathbb{R}$.
} 


\begin{tabular}{|c|c|c|c|c|c|}
\hline Formula & $z_{\mathrm{c}}^{\text {Dir }}(n=1)$ & $z_{\mathrm{c}}^{\text {Dir }}(n=2)$ & $z_{\mathrm{c}}^{\text {Dir }}(n=3)$ & $z_{\mathrm{c}}^{\text {Dir }}(n=4)$ & $z_{\mathrm{c}}^{\text {Dir }}(n=5)$ \\
\hline Analytical (eq. (5.5)) & $9.947 \times 10^{-3}$ & $4.671 \times 10^{-4}$ & $2.226 \times 10^{-5}$ & $1.061 \times 10^{-6}$ & $5.061 \times 10^{-8}$ \\
Numerical (eq. (3.12)) & $1.007 \times 10^{-2}$ & $4.673 \times 10^{-4}$ & $2.228 \times 10^{-5}$ & $1.062 \times 10^{-6}$ & $5.061 \times 10^{-8}$ \\
\hline
\end{tabular}

Table 3. Spinning exotic compact objects with reflective Dirichlet boundary conditions. We present the analytically calculated discrete set of dimensionless radii $z_{\mathrm{c}}^{\text {analytical }}(n)$ which characterize the spinning compact objects that can support the static (marginally-stable) massless scalar field configurations. We also present the corresponding radii $z_{\mathrm{c}}^{\text {numerical }}(n)$ of the horizonless compact objects as obtained from a direct numerical solution of the characteristic resonance condition (3.12). The data presented is for horizonless compact objects with dimensionless rotation parameter $\bar{a}=0.9$ linearly coupled to a massless scalar field mode with $l=m=1$. In the physically interesting regime $z_{\mathrm{c}} \ll 1$ of highly compact exotic objects, one finds a remarkably good agreement between the approximated radii $\left\{z_{\mathrm{c}}^{\text {analytical }}(n)\right\}$ of the compact objects that can support the marginally-stable scalar resonances (as calculated from the analytical resonance spectrum (5.5)) and the corresponding exact radii $\left\{z_{\mathrm{c}}^{\text {numerical }}(n)\right\}$ of the compact exotic objects (as determined by a direct numerical solution of the resonance equation (3.12)).

\begin{tabular}{|c|c|c|c|c|c|}
\hline Formula & $z_{\mathrm{c}}^{\mathrm{Neu}}(n=1)$ & $z_{\mathrm{c}}^{\mathrm{Neu}}(n=2)$ & $z_{\mathrm{c}}^{\mathrm{Neu}}(n=3)$ & $z_{\mathrm{c}}^{\mathrm{Neu}}(n=4)$ & $z_{\mathrm{c}}^{\mathrm{Neu}}(n=5)$ \\
\hline Analytical (eq. (5.6)) & $4.839 \times 10^{-2}$ & $2.145 \times 10^{-3}$ & $1.019 \times 10^{-4}$ & $4.860 \times 10^{-6}$ & $2.318 \times 10^{-7}$ \\
Numerical (eq. (3.13)) & $5.432 \times 10^{-2}$ & $2.153 \times 10^{-3}$ & $1.020 \times 10^{-4}$ & $4.860 \times 10^{-6}$ & $2.318 \times 10^{-7}$ \\
\hline
\end{tabular}

Table 4. Spinning exotic compact objects with reflective Neumann boundary conditions. We use the same physical parameters as in table 3 . In the physically interesting $z_{\mathrm{c}} \ll 1$ regime of highly compact exotic objects, one finds a remarkably good agreement between the approximated radii $\left\{z_{c}^{\text {analytical }}(n)\right\}$ of the compact exotic objects (see (5.6)) and the corresponding exact radii $\left\{z_{\mathrm{c}}^{\text {numerical }}(n)\right\}$ of the compact objects (as determined numerically from eq. (3.13)).

of the spinning exotic compact objects with reflecting Dirichlet/Neumann boundary conditions as calculated from the analytically derived resonance spectra (5.5) and (5.6). For comparison, we also display the corresponding radii $z_{\mathrm{c}}^{\text {numerical }}(n)$ of the horizonless compact objects as obtained from a direct numerical solution of the exact (analytically derived) resonance conditions (3.12) and (3.13).

From the data presented in tables 3 and 4 one finds a very good agreement, especially in the physically interesting regime $z_{\mathrm{c}} \ll 1$ of highly compact exotic objects ${ }^{15}$ (see eq. (2.2)), between the approximated radii of the horizonless compact objects that can support the static (marginally-stable) scalar resonances (as calculated from the analytical formulas (5.5) and (5.6)) and the corresponding exact radii of the spinning compact objects (as determined numerically from the characteristic resonance conditions (3.12) and (3.13)).

\footnotetext{
${ }^{15}$ As emphasized above, the small- $x_{\mathrm{c}}$ regime (5.1) corresponds to the physically interesting family, that was recently studied numerically in [13] (see also [1-12]), of highly compact horizonless exotic objects whose quantum reflective surfaces are located very near the would-be classical black-hole horizons (see eqs. (2.2) and (2.3)).
} 


\begin{tabular}{|c|c|c|c|c|c|}
\hline$\delta \equiv\left(r_{\mathrm{c}}-r_{+}\right) / M$ & $10^{-5}$ & $10^{-4}$ & $10^{-3}$ & $10^{-2}$ & $10^{-1}$ \\
\hline $\bar{a}^{\text {analytical }}$ & 0.999 & 0.999 & 1.001 & 1.000 & 1.001 \\
\hline
\end{tabular}

Table 5. Marginally-stable spinning exotic compact objects. We display, for various values of the compactness parameter $\delta \equiv\left(r_{\mathrm{c}}^{\max }-r_{+}\right) / M$ introduced in [13], the dimensionless ratio $\bar{a}^{\text {analytical }}(\delta) / \bar{a}^{\text {numerical }}(\delta)$ between the analytically derived critical angular momentum which characterizes the marginally-stable spinning exotic compact object and the corresponding numerically computed [13] value of the critical rotation parameter. The data presented are for the fundamental $(n=1)$ resonances of the spinning exotic compact objects with reflecting Dirichlet boundary conditions and for a massless scalar field mode with $l=m=1$. One finds a remarkably good agreement between our analytical results and the corresponding numerical data of [13].

\section{Analytical vs. former numerical results}

It is physically important to compare our analytical results for the marginally-stable exotic compact objects with the corresponding numerical data published recently in the very interesting work of Maggio, Pani, and Ferrari [13]. In table 5 we display, for various values of the compactness parameter $\delta \equiv\left(r_{\mathrm{c}}^{\max }-r_{+}\right) / M^{16}$ introduced in [13], the dimensionless ratio $\bar{a}^{\text {analytical }}(\delta) / \bar{a}^{\text {numerical }}(\delta)$. Here $\left\{\bar{a}^{\text {analytical }}(\delta)\right\}$ are the analytically derived dimensionless angular momenta which characterize the critical (marginally-stable) spinning exotic compact objects, and $\left\{\bar{a}^{\text {numerical }}(\delta)\right\}$ are the corresponding numerically computed [13] values of the critical rotation parameter. Interestingly, from the data presented in table 5 one finds a remarkably good agreement between our analytical formulas and the corresponding numerical data of [13].

\section{Summary and discussion}

Horizonless exotic compact objects with reflective properties have recently attracted much attention from physicists as possible quantum-gravity alternatives to classical black-hole spacetimes (see [1-13] and references therein). In a physically important work, Maggio, Pani, and Ferrari [13] have recently studied numerically the stability properties of a family of horizonless spinning exotic compact objects which are characterized by curved spacetime geometries that modify the Kerr metric only at some microscopic scale around the would-be classical horizon.

The numerical analysis presented in [13] has demonstrated that horizonless spinning compact objects with reflective surfaces may become superradiantly unstable to scalar perturbation modes. Intriguingly, however, the results presented in [13] have revealed the important fact that the ergoregion instability shuts down if the quantum reflective surface of the horizonless compact object is located far enough from the would-be classical black-hole horizon. This highly interesting numerical result implies, in particular, that there exists a unique family of marginally-stable spinning exotic compact objects which determine the critical boundary between stable and unstable horizonless spinning configurations.

\footnotetext{
${ }^{16}$ Note that the dimensionless coordinate $\delta$ introduced in [13], which characterizes the radius of the horizonless reflecting exotic compact object, can be expressed in the form $\delta=\frac{x_{\mathrm{c}}}{1-x_{\mathrm{c}}} \cdot \frac{r_{+}-r_{-}}{M}$ (see eq. (3.4)).
} 
In the present paper we have used analytical techniques in order to explore the physical properties of the critical (marginally-stable) spinning exotic compact objects. These horizonless reflecting objects are characterized by their ability to support spatially regular static configurations of massless scalar fields in their exterior spacetime regions. The physical significance of this unique family of critical (marginally-stable) exotic compact objects stems from the fact that it marks the boundary between stable and unstable composed spinning-exotic-compact-object-massless-scalar-field configurations.

The main results derived in the present paper and their physical implications are as follows:

(1) We have proved that, for given angular harmonic indices $(l, m)$ of the massless scalar field mode, there exist two discrete spectra of radii, $\left\{r_{\mathrm{c}}^{\text {Dirichlet }}(\bar{a}, l, m ; n)\right\}_{n=1}^{n=\infty}$ and $\left\{r_{\mathrm{c}}^{\text {Neumann }}(\bar{a}, l, m ; n)\right\}_{n=1}^{n=\infty}$, which characterize the horizonless spinning compact objects with reflective boundary conditions that can support the spatially regular static (marginally-stable) scalar field configurations. In particular, we have shown that the analytically derived resonance equations (see eqs. (3.12) and (3.13))

$$
\begin{aligned}
{ }_{2} F_{1}\left(l+1-2 i \alpha, l+1 ; 2 l+2 ; 1-x_{\mathrm{c}}^{\text {Dirichlet }}\right) & =0 \\
\text { and } \frac{d}{d x}\left[x^{-i \alpha}(1-x)^{l+1}{ }_{2} F_{1}(l+1-2 i \alpha, l+1 ; 2 l+2 ; 1-x)\right]_{x=x_{\mathrm{c}}^{\text {Neumann }}} & =0
\end{aligned}
$$

determine the critical dimensionless radii of the marginally-stable spinning exotic compact objects.

(2) We have shown that the physical properties of the critical (marginally-stable) horizonless spinning objects can be studied analytically in the physically interesting regime $x_{\mathrm{c}} \ll 1$ which corresponds to highly compact exotic objects. In particular, using analytical techniques, we have derived the remarkably compact resonance formula (see eqs. (5.5) and (5.6))

$$
x_{\mathrm{c}}(\bar{a}, l, m ; n)=e^{-\pi(n+\epsilon) / \alpha} \times \mathcal{F} ; \quad n \in \mathbb{Z},
$$

where

$$
\mathcal{F}(\bar{a}, l, m)=\left[\frac{\Gamma(2 i \alpha) \Gamma(l+1-2 i \alpha)}{\Gamma(-2 i \alpha) \Gamma(l+1+2 i \alpha)}\right]^{1 / 2 i \alpha} ; \quad \epsilon= \begin{cases}\frac{1}{2} & \text { Dirichlet B.C. } \\ 0 & \text { Neumann B.C. }\end{cases}
$$

for the discrete spectra of dimensionless radii which characterize the spinning compact objects that can support the static (marginally-stable) massless scalar field configurations.

It is worth stressing the fact that the physical significance of the largest (outermost) radii $r_{\mathrm{c}}^{\max }(\bar{a}) \equiv \max _{n}\left\{r_{\mathrm{c}}(\bar{a} ; n)\right\}$ stems from the fact that these critical dimensionless radii mark the boundary between stable and unstable horizonless compact objects with reflecting boundary conditions. In particular, spinning exotic compact objects whose reflecting surfaces are located in the radial region $r_{\mathrm{c}}<r_{\mathrm{c}}^{\max }(\bar{a})$ are superradiantly unstable to scalar perturbation modes, whereas spinning exotic compact 
objects whose reflecting surfaces are located in the radial region $r_{\mathrm{c}}>r_{\mathrm{c}}^{\max }(\bar{a})$ are stable.

(3) It has been explicitly demonstrated (see tables 3 and 4) that the analytically derived resonance spectra (5.5) and (5.6), which determine the dimensionless discrete radii of the horizonless exotic objects that can support the static (marginally-stable) scalar resonances, agree in the physically interesting regime $x_{\mathrm{c}} \ll 1$ of highly-compact objects with the corresponding exact radii of the critical exotic compact objects (as determined numerically directly from the resonance equations (3.12) and (3.13)).

(4) It is worth pointing out that the compact resonance spectra (7.2), which characterize the marginally-stable horizonless configurations, can be further simplified in the slow rotation regime $\bar{a} \ll 1$. In particular, one finds from (7.2) and (7.3) the resonance spectra $^{17}$

$$
x_{\mathrm{c}}(\bar{a} \ll 1, l, m ; n)=e^{-2 \pi\left(n+\epsilon-\frac{1}{2}\right) / m \bar{a}} \times e^{-2[\psi(l+1)-\psi(1)]} ; \quad n \in \mathbb{Z}
$$

in the regime $\bar{a} \ll 1$ (or equivalently, $\alpha \simeq m \bar{a} / 2 \ll 1$, see eq. (3.4)) of slowly-rotating exotic compact objects. It is worth noting that this analytical formula is especially useful since it is highly difficult to probe numerically [13] the small $\bar{a} \ll 1$ regime (which, as is evident from (7.4), corresponds to exponentially small values of the dimensionless radius $\left.x_{\mathrm{c}}(\bar{a} \ll 1)\right)$.

(5) It is interesting to note that a no-scalar-hair theorem has recently been derived for spherically symmetric (non-rotating) horizonless compact reflecting stars [70]. This theorem [70] excludes, in particular, the existence of spatially regular configurations made of massless scalar fields non-linearly coupled to horizonless compact objects with reflecting boundary conditions. On the other hand, in the present paper we have explicitly established the existence of massless scalar field configurations linearly coupled to non-spherically symmetric (rotating) compact objects. It is worth noting that the analytically derived formula (7.4) agrees with these findings. In particular, one finds from (7.4) that there are no marginally stable $(\omega=0)$ scalar field configurations in the non-rotating $\bar{a}=0$ case (that is, $x_{\mathrm{c}}=0^{18}$ for non-rotating horizonless compact objects with $\bar{a}=0$ ).

(6) Finally, we have explicitly demonstrated that our analytical formulas agree remarkably well with the corresponding numerical data that recently appeared in the interesting work of Maggio, Pani, and Ferrari [13].

\footnotetext{
${ }^{17}$ Here we have used the relations $[\Gamma(2 i \alpha) / \Gamma(-2 i \alpha)]^{1 / 2 i \alpha}=e^{2 \psi(1)+\pi / 2 \alpha}[1+O(\alpha)]$ and $[\Gamma(l+1-2 i \alpha) / \Gamma(l+$ $1+2 i \alpha)]^{1 / 2 i \alpha}=e^{-2 \psi(l+1)}[1+O(\alpha)]$ in the $\alpha=(m \bar{a} / 2) \cdot\left[1+O\left(\bar{a}^{2}\right)\right] \ll 1$ regime, where $\psi(z)$ is the Psi (Digamma) function (see eq. (6.3.1) of [59]).

${ }^{18}$ Note that the relation $x_{\mathrm{c}}=0$ corresponds to the physically unacceptable case of an horizonless compact object whose reflecting surface exactly coincides with the absorbing horizon of a black-hole spacetime.
} 


\section{Acknowledgments}

This research is supported by the Carmel Science Foundation. I thank Yael Oren, Arbel M. Ongo, Ayelet B. Lata, and Alona B. Tea for stimulating discussions.

Open Access. This article is distributed under the terms of the Creative Commons Attribution License (CC-BY 4.0), which permits any use, distribution and reproduction in any medium, provided the original author(s) and source are credited.

\section{References}

[1] P.O. Mazur and E. Mottola, Gravitational condensate stars: an alternative to black holes, gr-qc/0109035 [INSPIRE].

[2] S.D. Mathur, The fuzzball proposal for black holes: an elementary review, Fortschr. Phys. 53 (2005) 793 [hep-th/0502050] [INSPIRE].

[3] C.B.M.H. Chirenti and L. Rezzolla, How to tell a gravastar from a black hole, Class. Quant. Grav. 24 (2007) 4191 [arXiv:0706.1513] [InSPIRE].

[4] K. Skenderis and M. Taylor, The fuzzball proposal for black holes, Phys. Rept. 467 (2008) 117 [arXiv:0804.0552] [INSPIRE].

[5] P. Pani, E. Berti, V. Cardoso, Y. Chen and R. Norte, Gravitational wave signatures of the absence of an event horizon: nonradial oscillations of a thin-shell gravastar, Phys. Rev. D 80 (2009) 124047 [arXiv: 0909. 0287] [INSPIRE].

[6] A. Almheiri, D. Marolf, J. Polchinski and J. Sully, Black holes: complementarity or firewalls?, JHEP 02 (2013) 062 [arXiv:1207.3123] [INSPIRE].

[7] V. Cardoso, L.C.B. Crispino, C.F.B. Macedo, H. Okawa and P. Pani, Light rings as observational evidence for event horizons: long-lived modes, ergoregions and nonlinear instabilities of ultracompact objects, Phys. Rev. D 90 (2014) 044069 [arXiv:1406.5510] [INSPIRE].

[8] M. Saravani, N. Afshordi and R.B. Mann, Empty black holes, firewalls and the origin of Bekenstein-Hawking entropy, Int. J. Mod. Phys. D 23 (2015) 1443007 [arXiv:1212.4176] [INSPIRE].

[9] C. Chirenti and L. Rezzolla, Did GW150914 produce a rotating gravastar?, Phys. Rev. D 94 (2016) 084016 [arXiv: 1602.08759] [INSPIRE].

[10] J. Abedi, H. Dykaar and N. Afshordi, Echoes from the abyss: evidence for Planck-scale structure at black hole horizons, arXiv:1612.00266 [INSPIRE].

[11] B. Holdom and J. Ren, Not quite a black hole, Phys. Rev. D 95 (2017) 084034 [arXiv: 1612.04889] [INSPIRE].

[12] C. Barceló, R. Carballo-Rubio and L.J. Garay, Gravitational wave echoes from macroscopic quantum gravity effects, JHEP 05 (2017) 054 [arXiv:1701.09156] [INSPIRE].

[13] E. Maggio, P. Pani and V. Ferrari, Exotic compact objects and how to quench their ergoregion instability, arXiv:1703.03696 [INSPIRE].

[14] J.L. Friedman, Ergosphere instability, Commun. Math. Phys. 63 (1978) 243. 
[15] R. Brito, V. Cardoso and P. Pani, Superradiance. Energy extraction, black-hole bombs and implications for astrophysics and particle physics, Lect. Notes Phys. 906 (2015) 1 [arXiv: 1501.06570] [INSPIRE].

[16] S. Hod, Stationary scalar clouds around rotating black holes, Phys. Rev. D 86 (2012) 104026 [Erratum ibid. D 86 (2012) 129902] [arXiv:1211.3202] [INSPIRE].

[17] S. Hod, Stationary resonances of rapidly-rotating Kerr black holes, Eur. Phys. J. C 73 (2013) 2378 [arXiv:1311.5298] [INSPIRE].

[18] S. Hod, Kerr-Newman black holes with stationary charged scalar clouds, Phys. Rev. D 90 (2014) 024051 [arXiv: 1406.1179] [InSPIRE].

[19] S. Hod, Rotating black holes can have short bristles, Phys. Lett. B 739 (2014) 196 [arXiv: 1411.2609] [INSPIRE].

[20] S. Hod, The large-mass limit of cloudy black holes, Class. Quant. Grav. 32 (2015) 134002 [arXiv: 1607.00003] [INSPIRE].

[21] S. Hod, Extremal Kerr-Newman black holes with extremely short charged scalar hair, Phys. Lett. B 751 (2015) 177 [inSPIRE].

[22] S. Hod, A no-short scalar hair theorem for rotating Kerr black holes, Class. Quant. Grav. 33 (2016) 114001 [INSPIRE].

[23] S. Hod, The superradiant instability regime of the spinning Kerr black hole, Phys. Lett. B 758 (2016) 181 [arXiv: 1606. 02306] [INSPIRE].

[24] S. Hod and O. Hod, Analytic treatment of the black-hole bomb, Phys. Rev. D 81 (2010) 061502(R) [arXiv:0910.0734] [InSPIRE].

[25] S. Hod, On the instability regime of the rotating Kerr spacetime to massive scalar perturbations, Phys. Lett. B 708 (2012) 320 [arXiv:1205.1872] [INSPIRE].

[26] S. Hod, Spinning Kerr black holes with stationary massive scalar clouds: the large-coupling regime, JHEP 01 (2017) 030 [arXiv:1612.00014] [INSPIRE].

[27] C.A.R. Herdeiro and E. Radu, Kerr black holes with scalar hair, Phys. Rev. Lett. 112 (2014) 221101 [arXiv:1403.2757] [INSPIRE].

[28] C.L. Benone, L.C.B. Crispino, C.A.R. Herdeiro and E. Radu, Kerr-Newman scalar clouds, Phys. Rev. D 90 (2014) 104024 [arXiv: 1409.1593] [INSPIRE].

[29] C.A.R. Herdeiro and E. Radu, Ergosurfaces for Kerr black holes with scalar hair, Phys. Rev. D 89 (2014) 124018 [arXiv: 1406.1225] [INSPIRE].

[30] C.A.R. Herdeiro and E. Radu, A new spin on black hole hair, Int. J. Mod. Phys. D 23 (2014) 1442014 [arXiv:1405.3696] [InSPIRE].

[31] Y. Brihaye, C.A.R. Herdeiro and E. Radu, Myers-Perry black holes with scalar hair and a mass gap, Phys. Lett. B 739 (2014) 1 [arXiv:1408.5581] [INSPIRE].

[32] J.C. Degollado and C.A.R. Herdeiro, Wiggly tails: a gravitational wave signature of massive fields around black holes, Phys. Rev. D 90 (2014) 065019 [arXiv:1408.2589] [INSPIRE].

[33] C.A.R. Herdeiro, E. Radu and H.F. Rúnarsson, Non-linear Q-clouds around Kerr black holes, Phys. Lett. B 739 (2014) 302 [arXiv:1409.2877] [INSPIRE].

[34] C.A.R. Herdeiro and E. Radu, Construction and physical properties of Kerr black holes with scalar hair, Class. Quant. Grav. 32 (2015) 144001 [arXiv:1501.04319] [INSPIRE]. 
[35] C.A.R. Herdeiro and E. Radu, Asymptotically flat black holes with scalar hair: a review, Int. J. Mod. Phys. D 24 (2015) 1542014 [arXiv:1504.08209] [INSPIRE].

[36] C.A.R. Herdeiro and E. Radu, How fast can a black hole rotate?, Int. J. Mod. Phys. D 24 (2015) 1544022 [arXiv: 1505.04189] [INSPIRE].

[37] P.V.P. Cunha, C.A.R. Herdeiro, E. Radu and H.F. Rúnarsson, Shadows of Kerr black holes with scalar hair, Phys. Rev. Lett. 115 (2015) 211102 [arXiv:1509.00021] [INSPIRE].

[38] B. Kleihaus, J. Kunz and S. Yazadjiev, Scalarized hairy black holes, Phys. Lett. B 744 (2015) 406 [arXiv: 1503.01672] [INSPIRE].

[39] C.A.R. Herdeiro, E. Radu and H.F. Rúnarsson, Kerr black holes with self-interacting scalar hair: hairier but not heavier, Phys. Rev. D 92 (2015) 084059 [arXiv:1509.02923] [INSPIRE].

[40] C.A.R. Herdeiro, J. Kunz, E. Radu and B. Subagyo, Myers-Perry black holes with scalar hair and a mass gap: unequal spins, Phys. Lett. B 748 (2015) 30 [arXiv: 1505.02407] [INSPIRE].

[41] C.A.R. Herdeiro, E. Radu and H.F. Rúnarsson, Kerr black holes with Proca hair, Class. Quant. Grav. 33 (2016) 154001 [arXiv: 1603.02687] [INSPIRE].

[42] C.A.R. Herdeiro, E. Radu and H.F. Rúnarsson, Spinning boson stars and Kerr black holes with scalar hair: the effect of self-interactions, Int. J. Mod. Phys. D 25 (2016) 1641014 [arXiv: 1604.06202] [INSPIRE].

[43] Y. Brihaye, C.A.R. Herdeiro and E. Radu, Inside black holes with synchronized hair, Phys. Lett. B 760 (2016) 279 [arXiv:1605.08901] [INSPIRE].

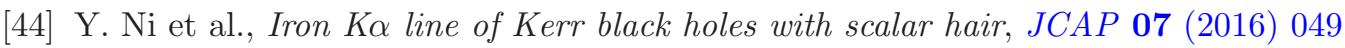
[arXiv: 1606.04654] [INSPIRE].

[45] M. Wang, Quantum and classical aspects of scalar and vector fields around black holes, arXiv: 1606.00811 [INSPIRE].

[46] S. Chandrasekhar, The mathematical theory of black holes, Oxford University Press, New York U.S.A. (1983).

[47] P. Pani, I-Love-Q relations for gravastars and the approach to the black-hole limit, Phys. Rev. D 92 (2015) 124030 [Erratum ibid. D 95 (2017) 049902] [arXiv:1506.06050] [INSPIRE].

[48] N. Uchikata, S. Yoshida and P. Pani, Tidal deformability and I-Love-Q relations for gravastars with polytropic thin shells, Phys. Rev. D 94 (2016) 064015 [arXiv:1607.03593] [INSPIRE].

[49] K. Yagi and N. Yunes, I-Love-Q anisotropically: universal relations for compact stars with scalar pressure anisotropy, Phys. Rev. D 91 (2015) 123008 [arXiv:1503.02726] [INSPIRE].

[50] S.A. Teukolsky, Rotating black holes: separable wave equations for gravitational and electromagnetic perturbations, Phys. Rev. Lett. 29 (1972) 1114 [INSPIRE].

[51] S.A. Teukolsky, Perturbations of a rotating black hole. I. Fundamental equations for gravitational electromagnetic and neutrino field perturbations, Astrophys. J. 185 (1973) 635 [INSPIRE].

[52] T. Hartman, W. Song and A. Strominger, Holographic derivation of Kerr-Newman scattering amplitudes for general charge and spin, JHEP 03 (2010) 118 [arXiv:0908.3909] [INSPIRE].

[53] A. Ronveaux, Heun's differential equations, Oxford University Press, Oxford U.K. (1995). 
[54] C. Flammer, Spheroidal wave functions, Stanford University Press, Stanford U.S.A. (1957).

[55] P.P. Fiziev, Classes of exact solutions to Regge-Wheeler and Teukolsky equations, arXiv:0902.1277 [INSPIRE].

[56] R.S. Borissov and P.P. Fiziev, Exact solutions of Teukolsky master equation with continuous spectrum, Bulg. J. Phys. 37 (2010) 065 [arXiv:0903.3617] [INSPIRE].

[57] P.P. Fiziev, Teukolsky-Starobinsky identities: a novel derivation and generalizations, Phys. Rev. D 80 (2009) 124001 [arXiv:0906.5108] [INSPIRE].

[58] P.P. Fiziev, Classes of exact solutions to the Teukolsky master equation, Class. Quant. Grav. 27 (2010) 135001 [arXiv:0908.4234] [INSPIRE].

[59] M. Abramowitz and I.A. Stegun, Handbook of mathematical functions, Dover Publications, New York U.S.A. (1970).

[60] S. Hod, Weak cosmic censorship: as strong as ever, Phys. Rev. Lett. 100 (2008) 121101 [arXiv: 0805.3873] [INSPIRE].

[61] S. Hod, Asymptotic spectrum of the oblate spin-weighted spheroidal harmonics: a WKB analysis, Phys. Lett. B 717 (2012) 462 [arXiv:1304.0529] [INSPIRE].

[62] S. Hod, Black-hole perturbation theory: the asymptotic spectrum of the prolate spin-weighted spheroidal harmonics, Phys. Rev. D 87 (2013) 064017 [arXiv:1304.4683] [INSPIRE].

[63] S. Hod, Eigenvalue spectrum of the spheroidal harmonics: a uniform asymptotic analysis, Phys. Lett. B 746 (2015) 365 [arXiv: 1506.04148] [InSPIRE].

[64] Y.B. Zel'dovich, Generation of waves by a rotating body, Pisma Zh. Eksp. Teor. Fiz. 14 (1971) 270 [JETP Lett. 14 (1971) 180].

[65] Y.B. Zel'dovich, Amplification of cylindrical electromagnetic waves reflected from a rotating body, Zh. Eksp. Teor. Fiz. 62 (1972) 2076 [Sov. Phys. JETP 35 (1972) 1085].

[66] W.H. Press and S.A. Teukolsky, Floating orbits, superradiant scattering and the black-hole bomb, Nature 238 (1972) 211 [INSPIRE].

[67] W.H. Press and S.A. Teukolsky, Perturbations of a rotating black hole. II. Dynamical stability of the Kerr metric, Astrophys. J. 185 (1973) 649 [INSPIRE].

[68] A.V. Vilenkin, Exponential amplification of waves in the gravitational field of ultrarelativistic rotating body, Phys. Lett. B 78 (1978) 301 [INSPIRE].

[69] P.M. Morse and H. Feshbach, Methods of theoretical physics, McGraw-Hill, New York U.S.A. (1953).

[70] S. Hod, No-scalar-hair theorem for spherically symmetric reflecting stars, Phys. Rev. D 94 (2016) 104073 [arXiv:1612.04823] [INSPIRE]. 